\title{
Practical Advances in Raman Microscopy
}

\author{
Thomas J Tague Jr and Jun Zhao
}

Bruker Optics, Inc., 19 Fortune Drive, Billerica, MA 01821

Previously, Raman microscopy required considerable expertise to conduct many tasks from simple routine acquisitions to confocal depth profiling. Calibration of wavelength axis has been problematic, where user intervention was required and had to be performed at least daily. The SureCal approach to wavelength calibration conducts the calibration automatically to better than $0.1 \mathrm{~cm}^{-1}$ accuracy and precision without the necessity of user calibrations. This patented method ${ }^{1}$ utilizes a Neon lamp that travels coaxial to the excitation laser and impinges onto the detector and is analogous to the HeNe laser reference used in FTIR spectrometers. Since the calibration is highly effective, single frequency or frequency stable lasers are no longer necessary for careful Raman spectroscopy, thereby significantly reducing the cost. Figure 1 shows the uncorrected laser wavelength in red and the corrected Raman shift in blue.

Another shortcoming to conducting routine Raman measurements is fluorescence. Many dyes and pigments exhibit fluorescence as well as textiles and active drug ingredients. For these cases, longer excitation wavelengths may be employed with limited success. The Raman scattering efficiency drops off as a fourth power as the wavelength is increased, thereby reducing sensitivity. A patented Automatic Fluorescence Rejection (AFR) method $^{2}$ has been used for rejecting fluorescence from many samples. The excitation laser is operated at two different temperatures or current to generate two spectra. These two spectra are subtracted one from another to generate the Raman difference spectrum, where the fluorescence is significantly rejected. The Raman difference spectrum is then integrated yielding the desired Raman spectrum without fluorescence as shown in Figures $2 \mathrm{a}$ and $2 \mathrm{~b}^{3}$.

\section{References:}

1. US Patent 6,141,095

2. US Patent $6,281,971$

3. Automated Fluorescence Rejection Using Shifted Excitation Raman Difference Spectroscopy, Zhao, J, Carraba, M, and Allen, F, Applied Spectroscopy Vol 56, No. 7, 2002. 


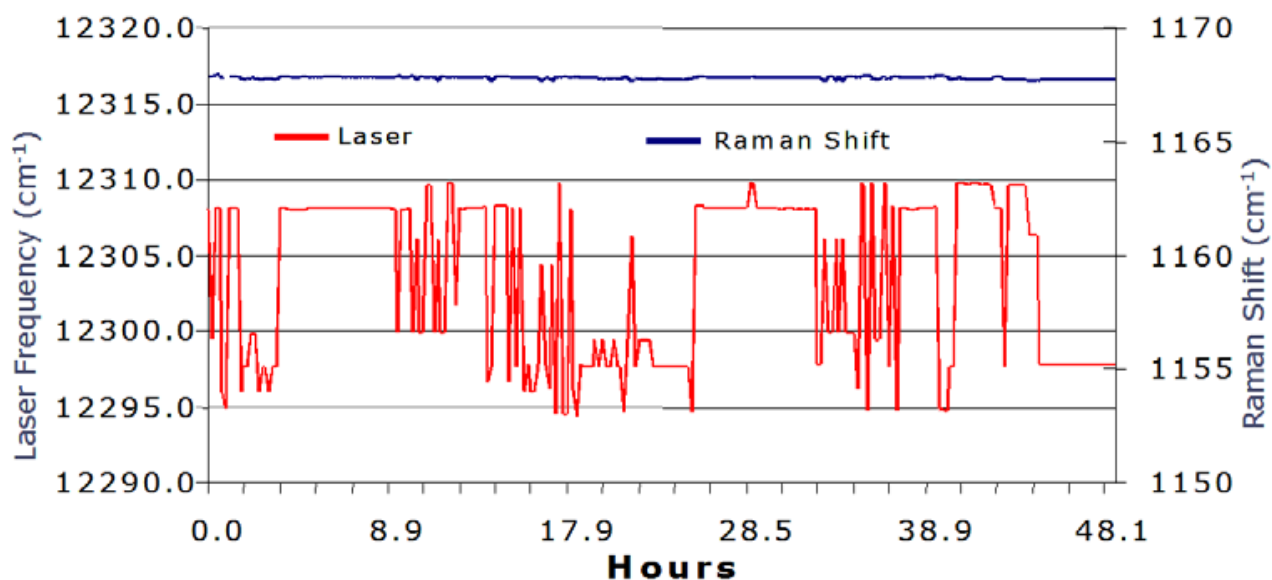

Figure 1 - Laser frequency (red) and Raman shift (blue) over time.

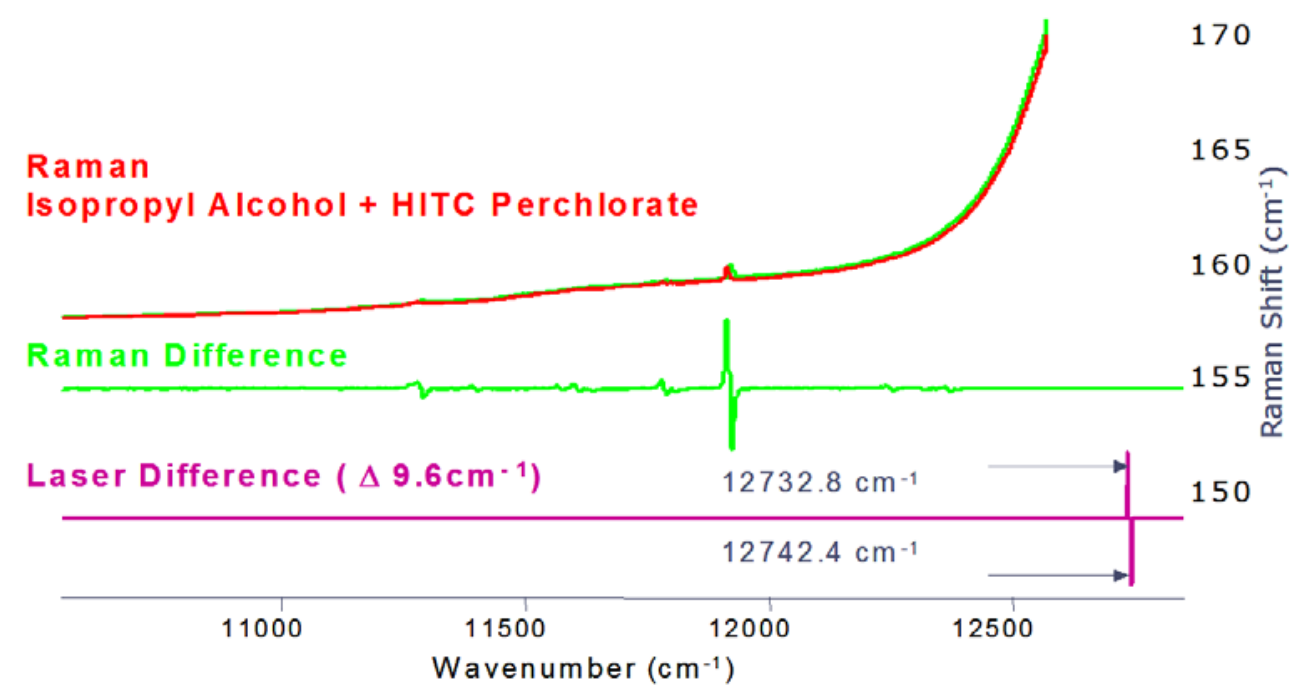

Figure $2 \mathrm{a}-$ Raman analysis of isopropanol with fluorescing HITC Perchlorate.

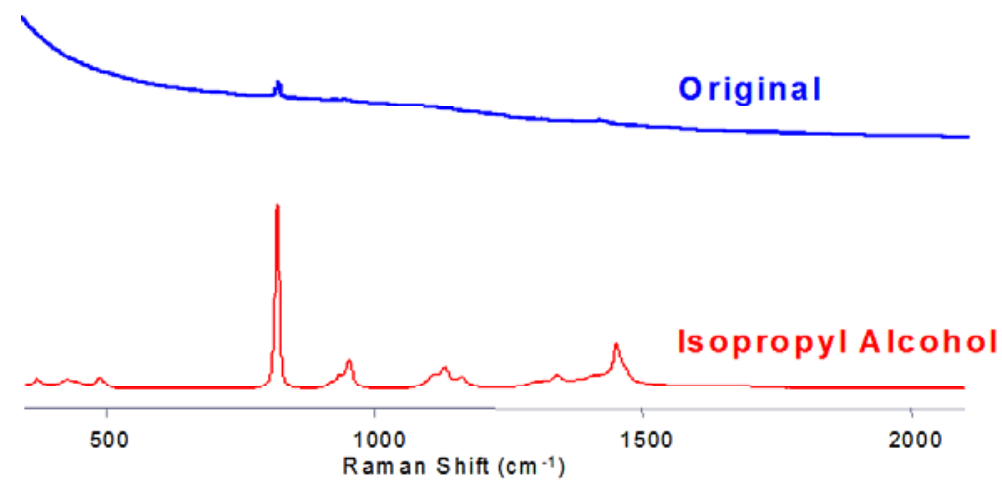

Figure $2 b$ - Resultant Raman spectrum of isopropanol after AFR method is applied. 